\title{
Revealing the cold dust in low-metallicity environments
}

\section{Photometry analysis of the Dwarf Galaxy Survey with Herschel (Corrigendum)}

\author{
A. Rémy-Ruyer ${ }^{1}$, S. C. Madden ${ }^{1}$, F. Galliano ${ }^{1}$, S. Hony ${ }^{1}$, M. Sauvage ${ }^{1}$, G. J. Bendo ${ }^{2}$, H. Roussel ${ }^{3}$, M. Pohlen ${ }^{4}$, \\ M. W. L. Smith ${ }^{4}$, M. Galametz ${ }^{5}$, D. Cormier ${ }^{6}$, V. Lebouteiller ${ }^{1}$, R. Wu ${ }^{1}$, M. Baes ${ }^{7}$, M. J. Barlow ${ }^{8}$, M. Boquien ${ }^{9}$, \\ A. Boselli ${ }^{10}$, L. Ciesla ${ }^{11}$, I. De Looze ${ }^{7}$, O. Ł. Karczewski ${ }^{12}$, P. Panuzzo ${ }^{13}$, L. Spinoglio ${ }^{14}$, M. Vaccari ${ }^{15}$, \\ C. D. Wilson ${ }^{16}$, and the Herschel-SAG2 consortium \\ ${ }^{1}$ Laboratoire AIM, CEA, Université Paris Sud XI, IRFU/Service d'Astrophysique, Bât. 709, 91191 Gif-sur-Yvette, France \\ e-mail: aurelie.remy@cea.fr \\ 2 UK ALMA Regional Centre Node, Jodrell Bank Centre for Astrophysics, School of Physics \& Astronomy, \\ University of Manchester, Oxford Road, Manchester M13 9PL, UK \\ 3 Institut d'Astrophysique de Paris, UMR 7095 CNRS, Université Pierre \& Marie Curie, 98bis boulevard Arago, 75014 Paris, France \\ 4 School of Physics \& Astronomy, Cardiff University, The Parade, Cardiff, CF24 3AA, UK \\ 5 European Southern Observatory, Karl-Schwarzschild-Str. 2, 85748 Garching-bei-München, Germany \\ ${ }^{6}$ Zentrum für Astronomie der Universität Heidelberg, Institut für Theoretische Astrophysik, Albert-Ueberle-Str. 2, \\ 69120 Heidelberg, Germany \\ 7 Sterrenkundig Observatorium, Universiteit Gent, Krijgslaan 281 S9, 9000 Gent, Belgium \\ 8 Department of Physics and Astronomy, University College London, Gower St, London WC1E 6BT, UK \\ 9 Institute of Astronomy, University of Cambridge, Madingley Road, Cambridge CB3 OHA, UK \\ ${ }^{10}$ Laboratoire d'Astrophysique de Marseille - LAM, Université d'Aix-Marseille \& CNRS, UMR 7326, 38 rue F. Joliot-Curie, \\ 13388 Marseille Cedex 13, France \\ 11 Department of Physics, University of Crete, 71003 Heraklion, Greece \\ 12 Department of Physics and Astronomy, University of Sussex, Brighton, BN1 9QH, UK \\ 13 GEPI, Observatoire de Paris, CNRS, Univ. Paris Diderot, Place Jules Janssen 92190 Meudon, France \\ 14 Instituto di Astrofisica e Planetologia Spaziali, INAF-IAPS, via Fosso del Cavaliere 100, 00133 Roma, Italy \\ 15 Physics Department, University of the Western Cape, Private Bag X17, 7535, Bellville, Cape Town, South Africa \\ 16 Department of Physics \& Astronomy, McMaster University, Hamilton Ontario L8S 4M1, Canada
}

A\&A 557, A95 (2013), DOI: 10.1051/0004-6361/201321602

Key words. galaxies: ISM - galaxies: dwarf - galaxies: photometry - infrared: galaxies - infrared: ISM - errata, addenda

\section{Introduction}

We present here the corrected results for the dust-to-stellar mass ratios versus metallicity for the two Herschel samples, the DGS and KINGFISH samples, after finding an error in the stellar masses for the DGS galaxies. We present corrected PACS upper limits for three galaxies and the correct value of the FIR luminosity of SBS0335-052. We also computed the stellar masses for KINGFISH using the same formula as for the DGS and look at $\beta=2.0$ modified blackbody dust masses to see how this influences the new derived relation between dust-to-stellar mass ratios and metallicity. We find that when the stellar masses are consistently estimated for both samples, the observed correlation between the dust-to-stellar mass ratios and metallicity is partly due to the choice of leaving the emissivity index free in the modified blackbody fits. Using $\beta=2.0$ to estimate the dust masses from a modified blackbody does not yield any clear dependence of the dust-to-stellar mass ratios on metallicity for these two samples.

\section{PACS photometry}

For three galaxies, HS $0822+3542$, HS $1442+4250$ and Tol 0618-402, some upper limits provided by
Rémy-Ruyer et al. (2013) are slightly too low and have been updated to the following values:

$$
\begin{aligned}
& \text { - HS 0822+3542: } F_{70} \leq 0.025 \mathrm{Jy} \text { and } F_{100} \leq 0.023 \mathrm{Jy} \text {, } \\
& \text { - HS 1442+4250: } F_{100} \leq 0.054 \mathrm{Jy} \text {, and } \\
& \text { - Tol0618-402: } F_{100} \leq 0.011 \mathrm{Jy} \text {. }
\end{aligned}
$$

None of these modifications affect the results of the paper because these galaxies are not detected in most Herschel bands, and thus have not been modelled with the modified blackbody model used in Rémy-Ruyer et al. (2013).

\section{Dust-to-stellar mass ratios}

The stellar masses used by Rémy-Ruyer et al. (2013) for the Dwarf Galaxy Survey (DGS), originally from Madden et al. (2013) were accidentally incorrect, and the right stellar masses for the DGS are presented now in Madden et al. (2014). We therefore present and analyse here the new plot of $M_{\mathrm{BB}} / M_{\mathrm{star}}$ as a function of metallicity for the DGS and KINGFISH samples (Fig. 1, top left).

The stellar masses for KINGFISH can be found in Skibba et al. (2011) and the DGS stellar masses in Madden et al. (2014). 

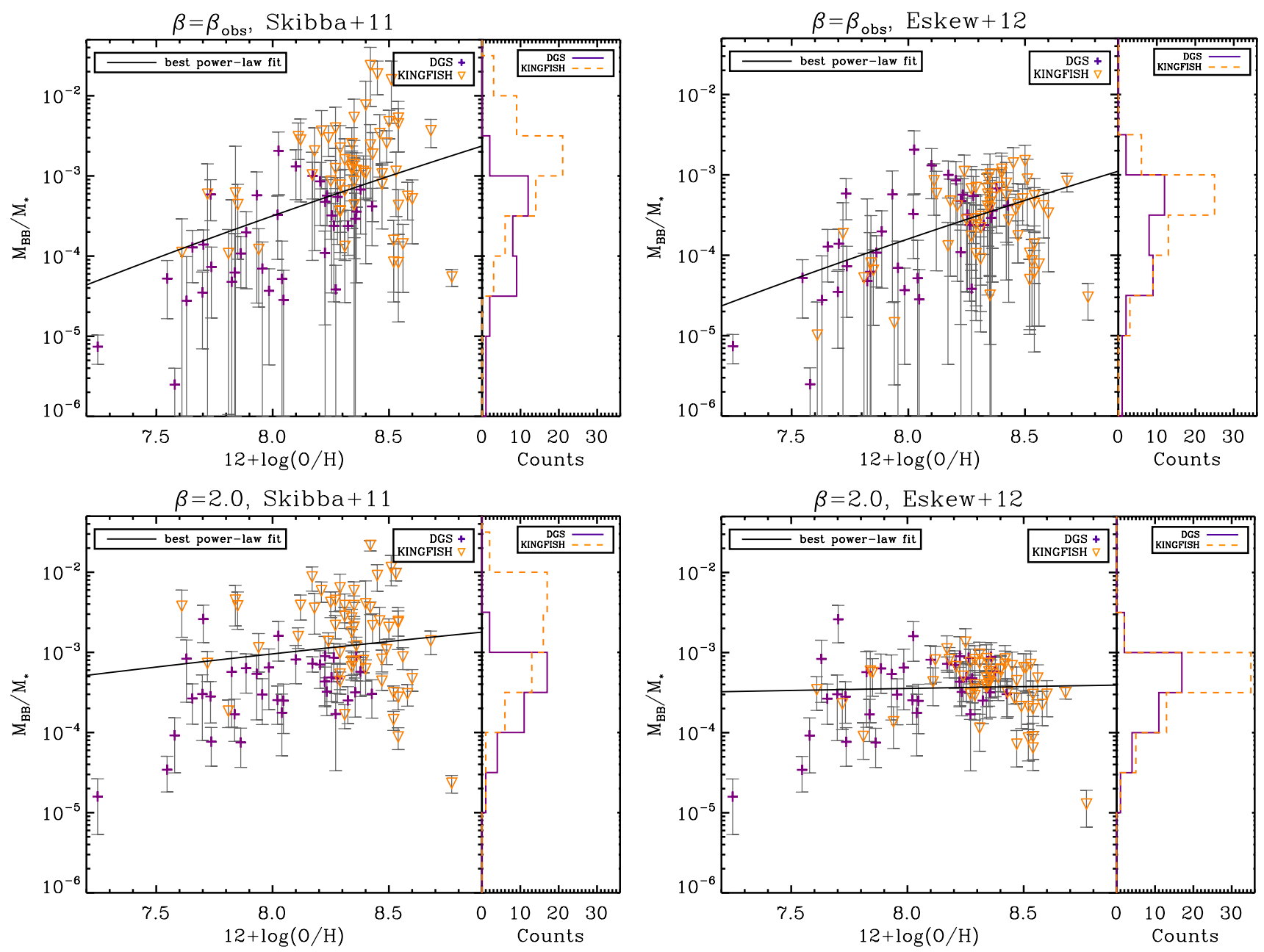

Fig. 1. $M_{\mathrm{BB}} / M_{\star}$ as a function of metallicity for DGS (purple crosses) and KINGFISH (orange downward triangles). The best power-law fit is indicated as a black line. The distribution of $M_{\mathrm{BB}} / M_{\star}$ is indicated on the side for both samples: plain purple line for DGS and dashed orange line for KINGFISH. The errors on the metallicities are omitted for clarity. They are about 0.1 dex on average. Top left: the dust masses are from a modified blackbody fit with a free emissivity index, $\beta_{\text {obs }}$ (Rémy-Ruyer et al. 2013), and the KINGFISH stellar masses are from Skibba et al. (2011). The best fit line corresponds to $\log \left(M_{\mathrm{BB}} / M_{\star}\right)=(-20.5 \pm 1.5)+(18.8 \pm 1.6) \times \log (12+\log (\mathrm{O} / \mathrm{H}))$. Top right: the dust masses are from a modified blackbody fit with a free emissivity index, $\beta_{\text {obs }}$ (Rémy-Ruyer et al. 2013), and the KINGFISH stellar masses are estimated with the formula of Eskew et al. (2012). The best fit line corresponds to $\log \left(M_{\mathrm{BB}} / M_{\star}\right)=(-20.2 \pm 1.6)+(18.2 \pm 1.7) \times \log (12+\log (\mathrm{O} / \mathrm{H}))$. Bottom left: the dust masses are from a modified blackbody fit with a fixed emissivity index, $\beta=2.0$, and the KINGFISH stellar masses are from Skibba et al. (2011). The best fit line corresponds to $\log \left(M_{\mathrm{BB}} / M_{\star}\right)=(-8.3 \pm 1.1)+(5.9 \pm 1.2) \times \log (12+\log (\mathrm{O} / \mathrm{H}))$. Bottom right: the dust masses are from a modified blackbody fit with a fixed emissivity index, $\beta=2.0$, and the KINGFISH stellar masses are estimated with the formula of Eskew et al. (2012). The best fit line corresponds to $\log \left(M_{\mathrm{BB}} / M_{\star}\right)=(-4.3 \pm 1.2)+(0.9 \pm 1.4) \times \log (12+\log (\mathrm{O} / \mathrm{H}))$. This figure replaces Fig. 14 (top panel) in Rémy-Ruyer et al. (2013).

Figure 1 (top left) shows that there is a strong decrease (slightly more than an order of magnitude) in the proportion of dust mass relative to the stellar mass with decreasing metallicity: we have a Spearman rank coefficient ${ }^{1} \rho=0.41$. The median for the ratio $M_{\text {dust }, \mathrm{BB}} / M_{\star}$ is $0.02 \%$ for DGS and $0.12 \%$ for KINGFISH. The best power-law fit gives

$M_{\text {dust,BB }} / M_{\star}=3.1 \times 10^{-21} \times(12+\log (\mathrm{O} / \mathrm{H}))^{18.8}$.

The stellar masses from the DGS are derived from the formula of Eskew et al. (2012) from the IRAC 3.6 and $4.5 \mu \mathrm{m}$ broadband

\footnotetext{
1 The Spearman rank coefficient, $\rho$, indicates how well the relationship between $\mathrm{X}$ and $\mathrm{Y}$ can be described by a monotonic function: monotonically increasing: $\rho>0$, or monotonically decreasing: $\rho<0$. For our number of sources $(\geq 100)$, a Spearman rank coefficient $\geq 0.30$ (or $\leq-0.30$ ) is the sign of a significant correlation between $\mathrm{X}$ and $\mathrm{Y}$ (i.e., the probability that the two variables are monotonically correlated is $\geq 99.9 \%$ ).
}

flux densities. The scatter in their relation corresponds to $1 \sigma$ uncertainties for their stellar masses of $\sim 30 \%$, which is within the uncertainties we have for the DGS stellar masses ( $~ 50 \%$ on average). The stellar masses for KINGFISH have been derived by Skibba et al. (2011) following Zibetti et al. (2009) from optical and NIR colours. With this estimate, the KINGFISH stellar masses could be biased in the low direction by up to $40 \%$ (Zibetti et al. 2009). We perform the test by computing the KINGFISH stellar masses with the formula by Eskew et al. (2012), using the IRAC flux densities of Dale et al. (2007), and find a much weaker correlation between the dust-to-stellar mass ratio and metallicity, $\rho=0.26$, and with a median $M_{\text {dust,BB }} / M_{\star}$ of $0.04 \%$ now for the KINGFISH sample (Fig. 1, top right).

The dust masses derived here for both samples, however, are probably lower limits of the real dust masses in many cases. In fact, we allow our $\beta_{\text {obs }}$ to go to very low values, giving lower dust masses than if we fixed it to 1.5 or even 2.0. Because we

\section{C1, page 2 of 3}


allow greater emission efficiency for the grains, we need less mass than if we were using a higher emissivity index to account for the same amount of luminosity. We perform the test by fixing the emissivity index parameter to 2.0 and find that the correlation between the dust-to-stellar mass ratios with metallicity vanishes. With $\beta=2.0$ modified blackbody dust masses, the Spearman rank coefficient for the relation decreases to $\rho=0.22$ if we are using Skibba et al. (2011) stellar masses for KINGFISH (Fig. 1 bottom left), and $\rho=2 \times 10^{-4}$ if we use the KINGFISH stellar masses derived from the formula of Eskew et al. (2012) (Fig. 1 bottom right).

As a conclusion, we find that when the stellar masses are estimated consistently for both samples using the formula by Eskew et al. (2012), the observed correlation between the dust-to-stellar mass ratios and metallicity is mostly due to the choice of leaving the emissivity index free in the modified blackbody fits. When $\beta=2.0$ is used to estimate the dust masses in the modified blackbody, no clear dependence of the dust-to-stellar mass ratios on metallicity can be observed. In a follow-up paper (Rémy-Ruyer et al., in prep.), we will obtain total dust masses from a full semiempirical SED model, which will allow us to study this parameter in more detail.

\section{Far-infrared luminosity to dust mass ratio}

We also found a typo for the far-infrared (FIR) luminosity of SBS0335-052, which should be $2.0_{-0.12}^{+0.14} \times 10^{8} L_{\odot}$, and not $1.2 \times$ $10^{7} L_{\odot}$ as quoted in Table 4 of Rémy-Ruyer et al. (2013). This FIR luminosity is computed between $50 \mu \mathrm{m}$ and $650 \mu \mathrm{m}$ (see the definition adopted by Rémy-Ruyer et al. 2013), and we might miss some luminosity at shorter wavelengths, since the SED peaks around $30 \mu \mathrm{m}$ in this galaxy (see Fig. A.1 in Rémy-Ruyer et al. 2013). In Fig. 2, we present the corrected point for SBS0335-052. Because this galaxy is our lowest metallicity galaxy, this new value has an influence on the derived bestfit relation. We now have a Spearman's rank coefficient of -0.74 ( -0.72 in Rémy-Ruyer et al. 2013) and the best power-law fit now gives

$L_{\mathrm{FIR}} / M_{\text {dust } \mathrm{BB}}=9.9 \times 10^{30} \times(12+\log (\mathrm{O} / \mathrm{H}))^{-30.5}$.

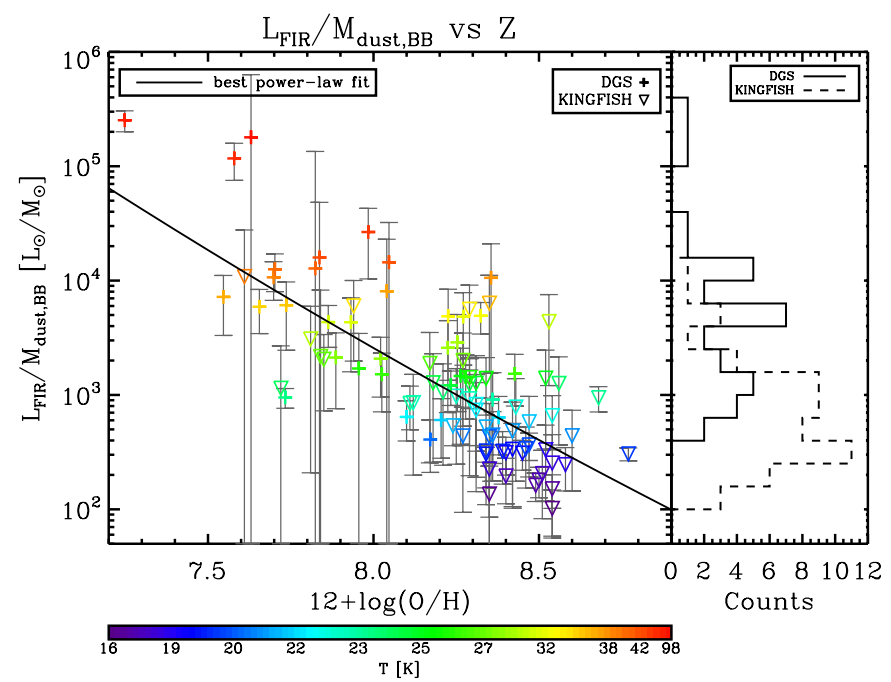

Fig. 2. $L_{\mathrm{FIR}} / M_{\mathrm{BB}}$ as a function of metallicity for DGS (crosses) and KINGFISH (downward triangles). The colours code the temperature, $T$ (Rémy-Ruyer et al. 2013). The best power-law fit line is indicated as a black line and corresponds to $\log \left(L_{\mathrm{FIR}} / M_{\mathrm{BB}}\right)=(31.0 \pm 0.9)+(-30.5 \pm$ $0.9) \times \log (12+\log (\mathrm{O} / \mathrm{H}))$. The distribution of $L_{\mathrm{FIR}} / M_{\mathrm{BB}}$ is indicated on the side for both samples: solid line for DGS and dashed line for KINGFISH. The errors on the metallicities are omitted for clarity. They are about 0.1 dex on average. This figure replaces Fig. 14 (bottom panel) in Rémy-Ruyer et al. (2013).

Our conclusions for the variation in the $L_{\mathrm{FIR}} / M_{\mathrm{dust}, \mathrm{BB}}$ ratios between the DGS and KINGFISH sample as presented in Rémy-Ruyer et al. (2013) are not affected.

\section{References}

Dale, D. A., Gil de Paz, A., Gordon, K. D., et al. 2007, ApJ, 655, 863 Eskew, M., Zaritsky, D., \& Meidt, S. 2012, AJ, 143, 139

Madden, S. C., Rémy-Ruyer, A., Galametz, M., et al. 2013, PASP, 125, 600

Madden, S. C., Rémy-Ruyer, A., Galametz, M., et al. 2014, PASP, 126, 1079 Rémy-Ruyer, A., Madden, S. C., Galliano, F., et al. 2013, A\&A, 557, A95 Skibba, R. A., Engelbracht, C. W., Dale, D., et al. 2011, ApJ, 738, 89

Zibetti, S., Charlot, S., \& Rix, H.-W. 2009, MNRAS, 400, 1181 